\title{
ON PURSUIT CURVES
}

\author{
J. C. BARTON ${ }^{1}$ and C. J. ELIEZER ${ }^{2}$
}

(Received 1 July 1996)

\section{Introduction}

Recently, several papers $[2-4,6]$ have been published concerning a pursuit problem which was apparently first posed explicitly by Leonardo da Vinci and which may have been present in earlier thinking about kinematics and geometry. Falconry appears to go back, in Europe, to the days of Pliny, Aristotle and Martial, and, in Asia, to 2000 $\mathrm{BC}[5]$.

We give here a brief survey of these papers and add some results for pursuit and flight in three dimensions, results which, although elementary, may not yet have been brought to notice.

George Boole [1] says that "the term curve of pursuit is given to the path which a point describes when moving with uniform velocity towards another point which moves with uniform velocity in a given curve".

Nowadays, since the advent of vector analysis, we would be more inclined to replace the word "velocity" by "speed". It is implicit in Boole's analysis that he is dealing with motion in two dimensions only.

Boole derives a second-order differential equation in plane coordinates $x, y$ for pursuit and flight in a plane and, by way of example, solves the equation for the case when the pursued point traverses the line $x=a$, beginning at time zero from the point $(a, 0)$, and the pursuing point begins the chase from the origin. When the speed ratio is 1 (pursuer and pursued moving with equal speeds) Boole finds the pursuit curve to be a parabola, a result which is clearly wrong since it is physically evident that the line $x=a$ must be an asymptote to the curve.

Colman [2] resurrected this problem, but in a slightly more general form in which the line $x=a$ is replaced essentially by the straight line $y=(x-a) \cot \alpha$ for $-\pi / 2<$ $\alpha<\pi / 2$. He does not give an explicit rectangular cartesian formula-equation for the

\footnotetext{
'North Carlton, VIC 3054, Australia.

${ }^{2}$ School of Mathematics, La Trobe University, Bundoora, VIC 3084, Australia.

(C) Australian Mathematical Society 2000, Serial-fee code $0334-2700 / 00$
} 
flight path of the pursuer but finds coordinates for the point of capture in the case when the speed ratio $c>1$. (Speed ratio $c$ is the ratio pursuer's speed : pursued's speed.)

It might be expected that, by choosing axes and origin suitably, this 'Colman' problem could be presented as a minor variation of Boole's example in which the pursuer begins at some point other than the origin; the authors have done this [4] and given an explicit cartesian equation for the pursuit curve. We give another method of deriving this path-formula below.

Guha and Biswas [6] showed how the 'Colman' problem could be analysed so as to bypass much of the heavy algebra that is evident in Colman's treatment.

\section{Flight and pursuit in three dimensions}

We give now differential equations for pursuit curves in three dimensions and exhibit some special solutions of them. The merit of doing this lies in the following consideration.

In the sense that simply expressed differential equations for such motions can be derived, the whole matter is thereby closed, for any pursuit problem becomes merely the problem of solving the differential equations subject to the appropriate starting conditions. Assuming that there will always be a computer which can solve the differential equations to any nominated degree of accuracy (in a step-by-step way), such elementary solutions as there are remain hidden, and it seems inherently valuable to bring as many of them as we can discover up to the light. This we do. As well, dropping one dimension to return to plane motion, we show how these differential equations can be solved to give an alternative solution of the 'Colman' problem.

Let the pursuer have rectangular coordinates $x, y, z$ in three dimensional space, and the pursued have coordinates $X, Y, Z$. The pursued always lies on the tangent, at $(x, y, z)$, to the pursuer's curve.

A tangent vector to the pursuer's curve has components $(\dot{x}, \dot{y}, \dot{z})$, dots denoting derivatives with respect to some parameter, for example time $t$, in terms of which the coordinates are expressible. Hence

$$
X=x+\lambda \dot{x}, \quad Y=y+\lambda \dot{y}, \quad Z=z+\lambda \dot{z},
$$

for some multiplier $\lambda$, which, in general, will depend on the parameter $t$.

As Boole observes, "the velocities of the two points being uniform, the corresponding elementary arcs will be in the constant ratio of the velocities with which they are described".

So we have by Pythagoras's theorem,

$$
\dot{x}^{2}+\dot{y}^{2}+\dot{z}^{2}=c^{2}\left(\dot{X}^{2}+\dot{Y}^{2}+\dot{Z}^{2}\right), \quad c \text { constant. }
$$


Equations (1) and (2) are four first-order, non-linear differential equations for the four functions $x(t), y(t), z(t), \lambda(t)$, where we suppose that the functions $X(t), Y(t), Z(t)$ are given.

We remark here that it is by no means necessary that pursuer and pursued traverse their paths with uniform speeds. All that is needed is that the speed ratio $c$ be constant, and (2) expresses this even for an arbitrary parameter $t$ which need not be a measure of elapsed time.

The differential equations are non-linear because of the Pythagorean metric of our space. We can not expect to obtain much, if anything, in the way of a general solution of them. We exhibit a few special solutions.

One of the simplest is a pair of circular cylindrical helices (screw threads), of equal pitch, but different diameters. Take, with no loss of generality, radius one unit for the pursuer's helix, and let the pitch be $p$ :

$$
x=\cos t, \quad y=\sin t, \quad z=(p / 2 \pi) t .
$$

Then, from (1), we have, for the pursued's curve:

$$
X=\cos t-\lambda \sin t, \quad Y=\sin t+\lambda \cos t, \quad Z=(p / 2 \pi)(t+\lambda)
$$

and, taking $\lambda$ constant, setting $\cos \alpha=\left(1+\lambda^{2}\right)^{-1 / 2}$, these can be written

$$
\begin{gathered}
X=\left(1+\lambda^{2}\right)^{1 / 2} \cos (t+\alpha), \quad Y=\left(1+\lambda^{2}\right)^{1 / 2} \sin (t+\alpha), \\
Z=\frac{p}{2 \pi}(t+\alpha)+\frac{p}{2 \pi}(\lambda-\alpha),
\end{gathered}
$$

which is a circular cylindrical helix of radius $\left(1+\lambda^{2}\right)^{1 / 2}$ and pitch $p$.

If $s, S$ denote respectively arc length measures along the paths of pursuer and pursued, the speed ratio is $(d s / d t) /(d S / d t)$ and is easily calculated to be

$$
c=\left(\frac{1+p^{2} /\left(4 \pi^{2}\right)}{1+\lambda^{2}+p^{2} /\left(4 \pi^{2}\right)}\right)^{1 / 2},
$$

which is less than 1.

If we imagine that pursuer and pursued describe these curves at constant speeds, the chase continues forever, the separation $\lambda\left(1+p^{2} /\left(4 \pi^{2}\right)\right)^{1 / 2}$ remaining constant.

This example is an extension, into three dimensions, of the concentric circular motion in the plane which we gave in Eliezer and Barton [3, p. 184].

Since the speed ratio $c$ is necessarily less than 1 for these circular helices, we ask whether we might find solutions, with $c>1$, for a different kind of helix, in particular one whose orthogonal projection on the $x y$ plane is an equiangular spiral. We are looking for an extension of the plane motion in two congruent spirals which we gave in [3, Section 4, pp. 183-4]. 
So we begin with the pursuer:

$$
x=e^{-k t} \cos t, \quad y=e^{-k t} \sin t, \quad z=f(t),
$$

where $f$ is at present left unspecified. We shall call such a curve an equiangularspiral-helix, because its orthogonal projection on the $x y$ plane is the equiangular spiral $\rho=e^{-k t}$ in which $\rho, t$ are plane polar coordinates with the pole at the origin; $\rho=\left(x^{2}+y^{2}\right)^{1 / 2}$ is the radius vector and $t$ is the azimuth.

A tangent vector has components given by

$$
\left(e^{-k t}(-k \cos t-\sin t), e^{-k t}(-k \sin t+\cos t), f^{\prime}(t)\right) .
$$

The current coordinates for a point on the curve of the pursued are as follows, in which $\lambda$ may vary with the parameter $t$ :

$$
\begin{gathered}
X=e^{-k t}\{(1-\lambda k) \cos t-\lambda \sin t\}, \quad Y=e^{-k t}\{(1-\lambda k) \sin t+\lambda \cos t\}, \\
Z=f+\lambda f^{\prime} .
\end{gathered}
$$

Referring to Figure 1, these can be written as

$$
X=H e^{-k t} \cos (t+\alpha), \quad Y=H e^{-k t} \sin (t+\alpha), \quad Z=f+\lambda f^{\prime} .
$$

If we keep $\lambda$ constant, then $\alpha$ and $H$ are constants and the orthogonal projection of the curve on the $x y$ plane is an equiangular spiral congruent to that of the pursuer, for $\left(X^{2}+Y^{2}\right)^{1 / 2}=H e^{-k t}=e^{-k\left(t+t_{0}\right)}$, where the constant $t_{0}$ satisfies $H=e^{-k t_{0}}$.

We now have set up a new problem: can we find a function $f$ such that, $\lambda$ being constant, these six coordinates will satisfy the speed condition (2)?

Substituting the coordinate functions into (2) we have

$$
e^{-2 k t}\left(1+k^{2}\right)+f^{\prime 2}=c^{2}\left\{H^{2} e^{-2 k t}\left(1+k^{2}\right)+\left(f^{\prime}+\lambda f^{\prime \prime}\right)^{2}\right\}
$$

and this is a non-linear second-order differential equation for the required function $f$. We remove the exponential factors by defining a new function $g(t)$ by the equation $f(t)=e^{-k t} g(t)$. Then $f^{\prime}=e^{-k t}\left(g^{\prime}-k g\right), f^{\prime \prime}=e^{-k t}\left(g^{\prime \prime}-2 k g^{\prime}+k^{2} g\right)$, and if these be substituted into (3), the exponential factor $e^{-2 k t}$ can be cancelled out. The equation that remains is

$$
\begin{aligned}
1+k^{2}+\left(-k g+g^{\prime}\right)^{2}= & c^{2}\left\{H^{2}\left(1+k^{2}\right)\right. \\
& \left.+\left[-k(1-k \lambda) g+(1-2 k \lambda) g^{\prime}+\lambda g^{\prime \prime}\right]^{2}\right\} .
\end{aligned}
$$

This is again a second-order non-linear differential equation for $g$. We abandon any attempt to find a general solution; instead we exhibit a few special solutions for which the corresponding flight and pursuit curves have some interesting features. 


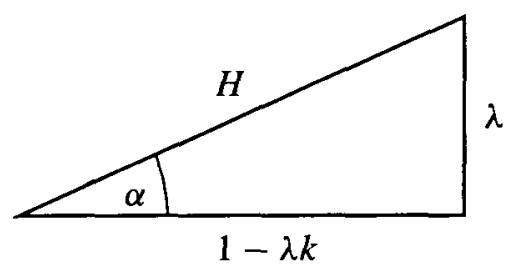

FIGURE 1.

The case of equal speeds: $c=1$. We take $k=1$ (the $45^{\circ}$ equiangular spiral) along with $c=1$ (equal speeds for pursuer and pursued) and $\lambda=1$. Consequently, $H=1$ and $\alpha=\pi / 2$ (Figure 1).

Equation (4) reduces to

$$
\left(g^{\prime}-g\right)^{2}=\left(-g^{\prime}+g^{\prime \prime}\right)^{2}
$$

so that

$$
g^{\prime}-g= \pm\left(-g^{\prime}+g^{\prime \prime}\right)
$$

We consider these two equations in turn.

(a) Taking the plus option:

$$
g^{\prime \prime}-2 g^{\prime}+g=0
$$

with solution

$$
g(t)=(A+B t) e^{t}
$$

and then

$$
f(t)=A+B t,
$$

where $A$ and $B$ are the arbitrary integration constants.

We can take $A$ zero, with no loss of generality, for this is just a matter of choosing the location of the origin on the $z$ axis. If then we take $B$ zero, the whole motion becomes a plane motion in the plane $z=0$ (see Figure 2).

$A P$ is the pursuer's path; $B Q$ is the pursued's path. The equations for $A P$ are

$$
x=e^{-t} \cos t, \quad y=e^{-t} \sin t, \quad z=0
$$




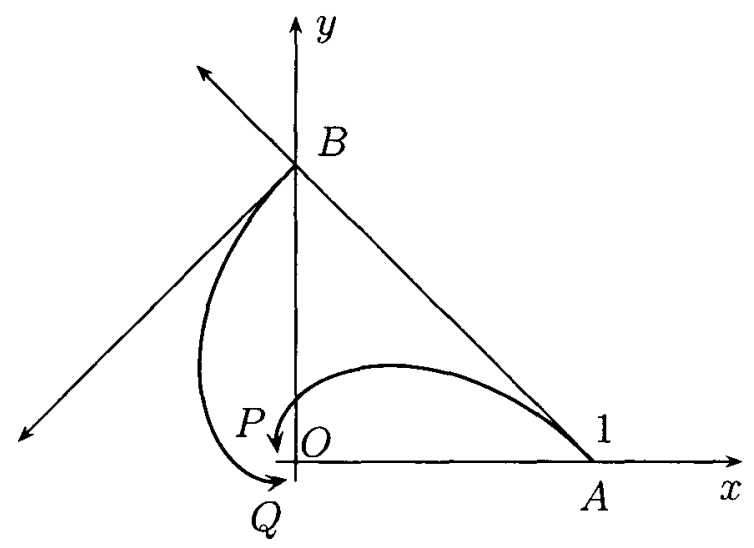

FIGURE 2.

and for $B Q$ are

$$
X=e^{-t} \cos (t+\pi / 2), \quad Y=e^{-t} \sin (t+\pi / 2), \quad Z=0 .
$$

Taking $B$ non-zero, positive say, the equations of the paths are

$$
\begin{array}{lll}
x=e^{-t} \cos t, & y=e^{-t} \sin t, & z=B t, \\
X=e^{-t} \cos (t+\pi / 2), & Y=e^{-t} \sin (t+\pi / 2), & Z=B(t+1) .
\end{array}
$$

The pursuer $P$ begins, at $t=0$, at the point $(1,0,0)$ and the pursued $Q$ begins at the point $(0,1, B)$. As the motion progresses, $P$ and $Q$ continue to climb and wind around the $z$ axis, getting very close to it but maintaining a separation just a little greater than, but approaching ever nearer to, $B$. Figure 2 shows the orthogonal projection, on the plane $z=0$, of the initial parts of the two flight paths.

(b) Taking the minus option in (5), we get $g^{\prime \prime}-g=0$ with solution $g(t)=$ $A e^{t}+B e^{-t}$. Consequently, $f(t)=A+B e^{-2 t}$.

As in (a), we can put $A$ zero without any effect on the shape or relative positions of the curves. Again, taking $B$ zero gives us motion in a plane.

If $B \neq 0$ and is positive say, $g(t)=B e^{-t}$ so that $f(t)=B e^{-2 t}$. The path equations are now as follows. For the pursuer:

$$
x=e^{-t} \cos t, \quad y=e^{-t} \sin t, \quad z=B e^{-2 t} \quad \text { for } t \geq 0 .
$$

The starting point is $(1,0, B)$.

For the pursued:

$$
X=e^{-t} \cos (t+\pi / 2), \quad Y=e^{-t} \sin (t+\pi / 2), \quad Z=-B e^{-2 t}
$$


for $t \geq 0$. The starting point is $(1,0,-B)$.

The pursuer spirals downwards from $(1,0, B)$ towards the origin while the pursued spirals upwards from $(1,0,-B)$ towards the origin. Again Figure 2 can be used as a representation of the orthogonal projection of the paths on to the $x y$ plane.

The motions we have obtained so far, for $c=1$, have been for the special conditions $k=\lambda=1$, and $g$ has been determined as an elementary function of $t$.

It is evident that there will be other elementary solutions, with $c=1$, in which we take $g$ constant. For in this case, (4) becomes

$$
k^{2} g^{2}(1-\lambda k)^{2}=k^{2} g^{2}-\left(H^{2}-1\right)\left(1+k^{2}\right)
$$

and there are positive values of $\lambda$ and $k$ satisfying this relation when $H^{2} \leq 1$. There are also solutions for $\lambda$ and $k$ for other values of $H$.

EXAMPLE. If we choose $k=1 / 2$ and $\lambda=2$, then $H=2, \alpha=\pi / 2$ and $g^{2}=15$. The pursuer has coordinates:

$$
x=e^{-t / 2} \cos t, \quad y=e^{-t / 2} \sin t, \quad z=\sqrt{15} e^{-t / 2}
$$

and starts, at $t=0$, from the point $(1,0, \sqrt{15})$.

The pursued moves in the plane $Z=0$ and has coordinates:

$$
X=2 e^{-t / 2} \cos (t+\pi / 2), \quad Y=2 e^{-t / 2} \sin (t+\pi / 2), \quad Z=0,
$$

and starts, at $t=0$, from the point $(0,2,0)$. The speeds are

$$
\begin{aligned}
& \sum \dot{x}^{2}=\left\{\left(\frac{1}{2}\right)^{2}+(1)^{2}+\frac{15}{4}\right\} e^{-t}=5 e^{-t}, \\
& \sum \dot{X}^{2}=\left\{4\left(\left(\frac{1}{2}\right)^{2}+(1)^{2}\right)+0\right\} e^{-t}=5 e^{-t} .
\end{aligned}
$$

Hence $c^{2}=\sum \dot{x}^{2} / \sum \dot{X}^{2}=1$.

This example will suffice to show that there will be a variety of such motions, depending on the choice of values for $k$ and $\lambda$.

We set aside further investigations of equal-speed paths to deal with those paths traversed with non-equal speeds. To keep this reasonably short we shall offer some numerical examples rather than essay a complete analysis. 
The case of non-equal speeds: $c \neq 1$. To get elementary solutions for $g$ from (4), we can, in the first instance, take $g$ constant; afterward we look at the case $g$ not constant.

(i) If $g$ is constant, so that $g^{\prime}=g^{\prime \prime}=0$, (4) reduces to

$$
1+k^{2}+k^{2} g^{2}=c^{2}\left\{H^{2}\left(1+k^{2}\right)+k^{2} g^{2}(1-k \lambda)^{2}\right\},
$$

which can be satisfied for some values of $c$ both greater than and less than 1 .

For example, let $\lambda=4$ and $k=1 / 2$, so that $H=\sqrt{17}$. Substituting these values into (6) we find

$$
5+g^{2}=c^{2}\left(85+g^{2}\right),
$$

and this relation can be satisfied for values of $c$ between $1 / \sqrt{17}$ and 1 by an appropriate choice of $g$.

We shall now give a numerical example in which $c>1$. Let $k=1$ and $\lambda=1$, so that $H=1$ and $\alpha=\pi / 2$. Let $g=2$. The pursuer has coordinates

$$
x=e^{-t} \cos t, \quad y=e^{-t} \sin t, \quad z=2 e^{-t}
$$

and starts, at $t=0$, from the point $(1,0,2)$.

The pursued has coordinates

$$
X=e^{-t} \cos (t+\pi / 2), \quad Y=e^{-t} \sin (t+\pi / 2), \quad Z=0,
$$

and starts from the point $(0,1,0)$.

We easily calculate the following:

$$
\sum \dot{x}^{2}=6 e^{-2 t}, \quad \sum \dot{X}^{2}=2 e^{-2 t}, \quad c^{2}=\frac{\sum \dot{x}^{2}}{\sum \dot{X}^{2}}=3, \quad \text { so } \quad c=\sqrt{3}>1 .
$$

Figure 2 again shows the orthogonal projection of the paths on to the $x y$ plane. The pursuer spirals down from $(1,0,2)$ towards the origin; the pursued spirals, in the plane $Z=0$, in towards the origin from the point $(0,1,0)$.

There is no capture despite the fact that the speed ratio is greater than 1 . The angle $\chi$ between the velocity vectors of pursuer and pursued can easily be calculated from the scalar product formula

$$
\cos \chi=\frac{\sum \dot{x} \dot{X}}{\left(\sum \dot{x}^{2} \sum \dot{X}^{2}\right)^{1 / 2}}
$$

and it is found that $\chi=\pi / 2$, independently of $t$, because $\sum \dot{x} \dot{X}$ is zero. 
The pursued is always moving at right angles to the line joining its position to that of the pursuer. There is a small paradox in that the path lengths, of both pursuer and pursued, calculated as limits $(t \rightarrow \infty)$, are finite; that if the speeds could be maintained constant, these paths would therefore be totally traversed in a finite time, that is they both terminate at the origin and yet there is no capture. It is the result of the infinite winding of the spirals.

(ii) Motions in which $g$ is not constant.

We can see that, if $c^{2} H^{2} \neq 1$, (4) will in general be a non-reducible quadratic equation in $g, g^{\prime}, g^{\prime \prime}$ and hence is most unlikely to yield elementary solutions for $g(t)$.

If $c^{2} H^{2}=1$, (4) is reducible to two linear equations in $g, g^{\prime}, g^{\prime \prime}$ whose coefficients are constant when $\lambda$ is constant. There will then be elementary exponential solutions for $g$. A further simplification is made by taking $\lambda k=1$.

Thus we put $H=\lambda=1 / c$ and $k=c$ so that (4) becomes

$$
\left(-c g+g^{\prime}\right)^{2}=c^{2}\left(-g^{\prime}+g^{\prime \prime} / c\right)^{2},
$$

equivalent to the two equations

$$
-c g+g^{\prime}= \pm\left(-c g^{\prime}+g^{\prime \prime}\right) .
$$

Taking the plus option, the differential equation has general solution

$$
g(t)=A e^{t}+B e^{c t} .
$$

Then

$$
f(t)=e^{-c t} g(t)=A e^{(1-c) t}+B .
$$

If $A=0$, the motion is entirely in the plane $z=B$, which we analysed in detail in [3].

Taking the minus option in (7), the differential equation has general solution

$$
g(t)=A e^{-t}+B e^{c t} .
$$

Then

$$
f(t)=e^{-c t} g(t)=A e^{(-1-c) t}+B .
$$

Similar remarks to those made about (8) apply to (9). We shall set $B=0$ without loss of generality and illustrate the case of non-zero $A$ by the following numerical example.

Take $c=2, k=2, \lambda=1 / 2, H=1 / 2$ and $\alpha=\pi / 2$. We first refer to the solution (8). The pursuer has coordinates

$$
x=e^{-2 t} \cos t, \quad y=e^{-2 t} \sin t, \quad z=A e^{-t} .
$$


The pursued has coordinates

$$
X=\frac{1}{2} e^{-2 t} \cos (t+\pi / 2), \quad Y=\frac{1}{2} e^{-2 t} \sin (t+\pi / 2), \quad Z=\frac{1}{2} A e^{-t} .
$$

We can easily calculate

$$
\begin{gathered}
\sum \dot{x}^{2}=5 e^{-4 t}+A^{2} e^{-2 t}, \quad \sum \dot{X}^{2}=\frac{5}{4} e^{-4 t}+\frac{1}{4} A^{2} e^{-2 t}, \\
c^{2}=\frac{\sum \dot{x}^{2}}{\sum \dot{X}^{2}}=4, \quad \text { as verification. }
\end{gathered}
$$

For $A>0$, in the first motion both pursuer and pursued are spiraling down towards the $x y$ plane; in the second motion the pursuer spirals downward and the pursued spirals upward.

There may be other real exponential solutions of (4), for the conditions $g$ not constant and $c^{2} H^{2}=1$, in which $\lambda k \neq 1$, but we shall not investigate this question. Whatever real solutions do exist will give motions that are similar to those we have just illustrated.

Instead we show how we can solve fairly simply the fundamental equations (1) and (2), with the third dimension suppressed, for the straight line flight case considered by Colman [2].

\section{The straight line problem revisited}

The pursued follows the straight line $A Q$ (Figure 3). The pursuer starts from $O$. The speed ratio is $c$, constant. We have to find the equation of the pursuer's path $O P$.

We may suppose that the speed of the pursued is 1 , so that, at time $t$, the coordinates of the pursued are $\left(t \cos \alpha, y_{0}+t \sin \alpha\right)$.

In the notation of (1) and (2), but with $z, Z$ removed, the equations to be solved for $x, y$ are

$$
\begin{aligned}
x+\lambda \dot{x} & =t \cos \alpha, \\
y+\lambda \dot{y} & =y_{0}+t \sin \alpha, \\
\dot{x}^{2}+\dot{y}^{2} & =c^{2} .
\end{aligned}
$$

We can simplify the right-hand sides of Equations (10)-(12) by choosing new axes $A X Y$ with the origin at $A, A Y$ along $A Q$ and $A X$ perpendicular to $A Q$ (see Figure 3).

Note that we have now abandoned the use of $X, Y$ to refer to the pursued, as in (1) and (2); $(X, Y)$ are now the coordinates of the pursuer referred to the new axes. 


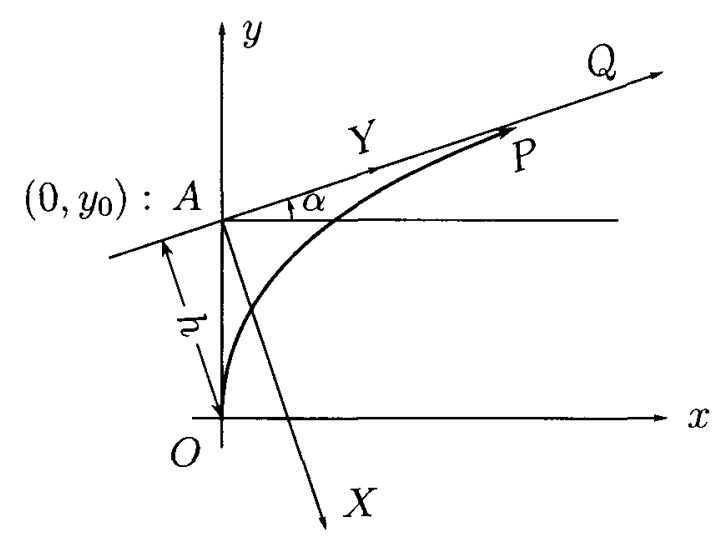

FIGURE 3.

The relations between $(x, y)$ and $(X, Y)$ are the standard ones for translation of origin and rotation of axes and one easily finds the new equations equivalent to (10)(12):

$$
\begin{aligned}
X+\lambda \dot{X} & =0 \\
Y+\lambda \dot{Y} & =t \\
\dot{X}^{2}+\dot{Y}^{2} & =c^{2}
\end{aligned}
$$

It is clear, physically, that $X$ is a one-way (decreasing) function of $t$, whence $t$ is a one-way function of $X$, and we choose to regard $X$ as the parameter, or independent variable, for the motion, and $\lambda, Y t$ as functions of $X$.

First we eliminate $\lambda$ from (13) and (14):

$$
\dot{X} Y-X \dot{Y}=t \dot{X}
$$

which we can write as

$$
Y-X\left(\frac{d Y}{d X}\right)=t
$$

Differentiating (16) with respect to $X$, we have

$$
-X\left(\frac{d^{2} Y}{d X^{2}}\right)=\frac{d t}{d X}
$$


Dividing (15) by $\dot{X}^{2}$ we get

$$
1+\left(\frac{d Y}{d X}\right)^{2}=c^{2}\left(\frac{d t}{d X}\right)^{2}
$$

Eliminating $d t / d X$ between (17) and (18) we find

$$
1+p^{2}=c^{2} X^{2}\left(\frac{d p}{d X}\right)^{2}
$$

where $p$ denotes $d Y / d X$.

Relative to the $X Y$ axes the pursuer's curve is concave upwards, that is, in the direction of the positive $Y$-axis, so that $d p / d X=d^{2} Y / d X^{2}$ is positive for all relevant $X$.

Taking the appropriate square root of (19) we get

$$
\left(1+p^{2}\right)^{1 / 2}=c X\left(\frac{d p}{d X}\right)
$$

and (2) is a simple separable variable differential equation from which we can determine $Y$ as a function of $X$, that is, the cartesian equation of the path of the pursuer.

The initial conditions at $t=0$ are, in terms of $X$ and $Y$,

$$
\begin{aligned}
\text { (i) } X & =h, \quad Y=-h \tan \alpha, \\
\text { (ii) } X & =h, \quad \frac{d Y}{d X}=\tan (\pi-\alpha)=-\tan \alpha,
\end{aligned}
$$

where we have written $h$ for $y_{0} \cos \alpha$. Integrating (20) once, we get

$$
\left\{\left(1+p^{2}\right)^{1 / 2}+p\right\}^{c}=\frac{X}{X_{0}}
$$

where $X_{0}$ is an integration constant. Using the initial condition (22), we find

$$
(\sec \alpha-\tan \alpha)^{c}=h / X_{0} .
$$

(The positive square root of $1+(-\tan \alpha)^{2}$ is $+\sec \alpha$ because $-\pi / 2<\alpha<\pi / 2$.) Hence

$$
X_{0}=h(\sec \alpha+\tan \alpha)^{c}
$$

and is now a known constant.

From (23) we have

$$
\left(1+p^{2}\right)^{1 / 2}+p=\left(\frac{X}{X_{0}}\right)^{1 / c}
$$


whence, taking reciprocals

$$
\left(1+p^{2}\right)^{1 / 2}-p=\left(\frac{X}{X_{0}}\right)^{-1 / c}
$$

and so

$$
p=\frac{d Y}{d X}=\frac{1}{2}\left\{\left(\frac{X}{X_{0}}\right)^{1 / c}-\left(\frac{X}{X_{0}}\right)^{-1 / c}\right\} .
$$

The integration of (25) is straightforward. An integral for the case $c=1$ will include a logarithm. We shall leave the details of this case to the reader, and briefly deal with the case $c \neq 1$.

An integral of $(25)$ is then

$$
\frac{Y}{X_{0}}-A=\frac{1}{2}\left\{\left(\frac{X}{X_{0}}\right)^{1+1 / c} \frac{c}{c+1}-\left(\frac{X}{X_{0}}\right)^{1-1 / c} \frac{c}{c-1}\right\},
$$

where $A$ is an integration constant.

Using the initial condition (21) $A$ can be evaluated:

$$
A=(\sec \alpha-\tan \alpha)^{c} \frac{c \sec \alpha+\tan \alpha}{c^{2}-1} .
$$

Now, inserting (24) and (27) into (26) we find, after tidying up, the required cartesian equation of the pursuer's path:

$$
\frac{Y}{h}=\frac{c \sec \alpha+\tan \alpha}{c^{2}-1}+\frac{1}{2}\left\{\frac{c}{c+1} w\left(\frac{X}{h}\right)^{1+1 / c}-\frac{c}{c-1} w^{-1}\left(\frac{X}{h}\right)^{1-1 / c}\right\},
$$

in which $w$ denotes $\sec \alpha-\tan \alpha$.

We expect that there will be capture when $c>1$ and no capture when $c<1$. A verification of this is found by calculating $\lambda$, which is a multiple, a constant one if we assume the speed to be constant, of the separation between pursuer and pursued.

From (13), (14) and (15) it is easy to verify that

$$
\lambda=\frac{X}{c}\left\{1+\left(\frac{d Y}{d X}\right)^{2}\right\}^{1 / 2}=\frac{X}{2 c}\left\{w\left(\frac{X}{h}\right)^{1 / c}+w^{-1}\left(\frac{X}{h}\right)^{-1 / c}\right\} .
$$

For $c>1, \lambda \rightarrow 0$ as $X \rightarrow 0$ and, in fact, $\lambda=0$ when $X=0$.

The coordinates of the point of capture are, from (28), $X=0, Y=h(c \sec \alpha+$ $\tan \alpha) /\left(c^{2}-1\right)$ and from these we find the $x$-coordinate to be

$$
x=Y \cos \alpha=y_{0} \frac{c \cos \alpha+\sin \alpha \cos \alpha}{c^{2}-1}
$$


as in Colman [2].

For $c<1, \lambda \rightarrow \infty$ and $Y \rightarrow \infty$ as $X \rightarrow 0$. The chase continues indefinitely, the pursuer lagging ever further behind. The line $A Q$ is an asymptote of the pursuer's curve.

\section{References}

[1] G. A. Boole, Treatise on differential equations (Cambridge, Macmillan and Co., 1859) p. 246.

[2] W. J. A. Colman, "A curve of pursuit", Bulletin of the Institute of Mathematics and its Applications 27 (3) (1991) pp. 45-47.

[3] C. J. Eliezer and J. C. Barton, "Pursuit curves", Bulletin of the Institute of Mathematics and its Applications $28(11,12)(1992)$ pp. 182-184.

[4] C. J. Eliezer and J. C. Barton, "Pursuit curves II", Bulletin of the Institute of Mathematics and its Applications $31(9,10)(1995)$ pp. 139-141.

[5] Encyclopaedia Britannica, Ninth Edition, vol. IX, MDCCCLXXIX, p. 6.

[6] A. Guha and S. K. Biswas, "On Leonardo da Vinci's cat and mouse problem", Bulletin of the Institute of Mathematics and its Applications $30(1,2)(1994)$ pp. 12-15. 